\title{
Avaliação dos sintomas oculares e impacto na qualidade de vida nos pacientes com o diagnóstico clínico de rosácea
}

\section{Lorraine S. Santos*, Andréa F. E. C França, Monica A. de Paula, Eduardo B. Barbosa, Dimitri F. Luz.}

\section{Resumo}

A Rosácea é uma dermatose inflamatória crônica comum e é classificada nas formas eritemato-telangiectásica, papulopustulosa, fimatosa e ocular. O presente trabalho tem como objetivo traçar o perfil epidemiológico dos pacientes com rosácea, atendidos nos ambulatórios de Oftalmologia e Dermatologia do HC- UNICAMP nos anos de 2018/2019, além de descrever os sinais e sintomas oftalmológicos através da avaliação da superfície ocular, filme lacrimal e glândulas de meibomius. Quarenta pacientes com rosácea foram selecionados quanto à gravidade da doença através do score clínico desenvolvido pelo National Rosacea Society Expert Comitee e submetidos ao DLQI (Dermatology Life Quality Index) e OSDI (Ocular Surface Disease Index).72\% mulheres, com idade média de 46 anos; 60\% dos pacientes pertenciam aos fototipos 1 e 2 de Fitzpatrick e metade deles estava sob tratamento tópico apenas. A forma eritematotelangectásia da rosácea foi a mais comum (56\%). O DLQI mostrou nenhum ou mínimo impacto na vida dos pacientes (DLQI $\leq 5)$, sem significância estatística com a gravidade da rosácea. As queixas de olho seco segundo o OSDI foram leves em $80 \%$ dos casos, mas a produção do filme lacrimal estava abaixo do normal em $48 \%$ deles, de acordo com o teste de Schirmer $(<10 \mathrm{~mm})$.

\section{Palavras-chave:}

Rosácea, sintomas oculares, qualidade de vida

\section{Introdução}

A rosácea é uma doença inflamatória crônica encontrada em $10 \%$ da população e classificada nas formas eritemato-telangiectásica, papulo-pustulosa, fimatosa, e ocular. A rosácea ocular ocorre em 58 a $72 \%$ dos pacientes, sendo que, em $20 \%$ dos casos, antecede as lesões de pele. Os achados mais comuns são blefarite, olho seco e conjuntivite. Devido à sintomatologia inespecífica, a rosácea ocular é frequentemente subdiagnosticada ${ }^{1}$. O presente trabalho tem como objetivo traçar o perfil epidemiológico dos pacientes com rosácea, atendidos nos ambulatórios de Oftalmologia e Dermatologia do HC- UNICAMP nos anos de 2018/2019, além de descrever os sinais e sintomas oftalmológicos através da avaliação da superfície ocular, filme lacrimal e glândulas de meibomius.

\section{Resultados e Discussão}

Estudo transversal, observacional e não intervencional. Quarenta pacientes com rosácea foram selecionados quanto à gravidade da doença através do score clínico desenvolvido pelo National Rosacea Society Expert Comitee e submetidos ao DLQI (Dermatology Life Quality Index) e OSDI (Ocular Surface Disease Index). Avaliações objetivas do filme lacrimal através do teste de Schirmer e meibografia foram realizadas pelo oftalmologista. Os resultados obtidos estão representados na suas tabelas a seguir.

Tabela 1. Dados clínico epidemiológicos dos pacientes com Rosácea.

\begin{tabular}{|c|c|}
\hline Parâmetro & $\mathrm{N}=40$ \\
\hline Idade (média $\pm D P$, anos) & $47 \pm 12$ \\
\hline $\operatorname{Sexo}(\mathrm{M} / \mathrm{F})$ & $11 / 29$ \\
\hline $\begin{array}{r}\text { Fototipo (\%) } \\
1 \text { e } 2 \\
3 \text { e } 4\end{array}$ & $\begin{array}{l}63 \\
37\end{array}$ \\
\hline $\begin{array}{l}\text { Grau de rosácea (número de pacientes) } \\
\text { Eritematotelangectásica } \\
\text { Papulopustulosa } \\
\text { Outros (fimatosa, ocular exclusiva) }\end{array}$ & $\begin{array}{l}22 \\
16 \\
6\end{array}$ \\
\hline DLQI (\%) & \\
\hline
\end{tabular}

\begin{tabular}{|l|l|}
\hline 0 a 1 (sem efeito na vida do paciente) & 24 \\
2 a 5 (pequeno efeito na vida do paciente) & 36 \\
6 a 10 (moderado efeito na vida do & 24 \\
paciente) & \\
$>10$ (grande efeito na vida do paciente) & 16 \\
\hline Tratamento atual (\%) & 17 \\
Nenhum & 51 \\
Tópico & 31 \\
Oral & \\
\hline
\end{tabular}

Tabela 2. Dados objetivos dos parâmetros oftalmológicos avaliados

\begin{tabular}{|l|l|}
\hline Parâmetro & \multicolumn{1}{|c|}{$\mathrm{N}=40$} \\
\hline OSDI (média) & 24,73 \\
\hline OSDI anormal $(\%)(>10)$ & $71,8 \%$ \\
\hline Schirmer teste (média, mm) & 13,67 \\
\hline Teste de Schirmer anormal $(\%)(<10 \mathrm{~mm})$ & $48,7 \%$ \\
\hline
\end{tabular}

Valor normal teste de Schirmer $>10 \mathrm{~mm}$ e Valor normal OSDI < 10

\section{Conclusões}

Idade, gênero, e fototipos aqui encontrados são similares aos dados americanos, a despeito de toda miscigenação brasileira. A qualidade de vida na rosácea é afetada independentemente da gravidade da doença cutânea. $\mathrm{O}$ estudo sugere que o comprometimento oftalmológico na rosácea não está relacionado diretamente à gravidade cutânea da doença e que a avaliação ocular objetiva pode estar alterada mesmo com mínimos sintomas. Cabe ao dermatologista suspeitar do dano ocular na rosácea a fim de estabelecer um diagnóstico precoce e evitar complicações futuras

1 Steinhoff, M. Schauber, J.; Leyden, J.J. New insights into rosacea pathophysiology: A review of recent findings. J. Am. Acad. Dermatol 2013, 69, S15-S26.

2 Two, A.M.; Wu, W.; Gallo, R.L.; Hata, T.R. Rosacea: Part I Introduction, categorization, histology, pathogenesis, and risk factors. J. Am. Acad. Dermatol. 2015, 72, 749-758.

3 Starr PA, McDonald A. Oculocutaneous aspects of rosacea. Proc R Soc Med. 1969; 62(1):9-11. 